\title{
CONVERGÊNCIA LOCAL DE RENDA NO BRASIL
}

\author{
Erika Cristina Barbosa de Almeida Ribeiro* \\ Eduardo Simões de Almeida ${ }^{\dagger}$
}

\begin{abstract}
Resumo
Diversos trabalhos verificam a hipótese de convergência de renda para as regiões brasileiras. Contudo, a persistência da desigualdade de renda ainda é verificada entre as regiões. Nesse contexto, utilizando a metodologia de Regressões Ponderadas Geograficamente (RPG), o presente trabalho busca analisar a hipótese de convergência local para as áreas mínimas comparáveis (AMCs). Os principais resultados confirmam a hipótese de múltiplos equilíbrios. Entretanto, de maneira mais sofisticada que a análise de clubes de convergência, os resultados da análise local indicam que cada AMC estaria convergindo para o seu específico estado estacionário.
\end{abstract}

Palavras-chave: Crescimento econômico; Convergência de Renda; Econometria Espacial.

\begin{abstract}
Several works verify the hypothesis of income convergence for Brazilian regions. However, income inequality's persistence can still be verified. In this context, using the methodology of Geographically Weighted Regression (GWR), this study aims to analyze the local income convergence among the Brazilians areas (AMCs). The main results indicate multiple equilibria hypotheses. But, in a more sophisticated manner than convergence clubs analysis, local analysis results indicate that each AMC would be converging to its specific steady state.
\end{abstract}

Keywords: Economic Growth, Income Convergence, Spatial Econometrics.

JEL classification: O40, R11, C31

\footnotetext{
* Universidade Federal de Juiz de Fora. E-mail: erika_almeidajf@yahoo.com.br

${ }^{\dagger}$ Universidade Federal de Juiz de Fora. E-mail: edualmei@gmail.com
} 


\section{Introdução}

A grande diferença de renda entre as economias intriga e faz surgir diversos estudos a respeito dos processos de crescimento e de convergência de renda entre as regiões. Desde os trabalhos de Baumol (1986) e Abramovitz (1986), que indicam a existência de convergência de renda per capita entre as regiões, tal análise passou por diversas sofisticações.

Em um trabalho posterior, Baumol e Wolff refinam a análise e encontram uma convergência de renda em clubes, ou seja, a influência da renda inicial per capita sobre as taxas de crescimento variaria de acordo com as características locais. Dessa forma, os autores concluem que cada clube apresentaria um steady state, onde regiões pertencentes ao mesmo clube convergiriam em renda entre si e que não haveria uma convergência de renda absoluta (Baumol \& Wolff 1988).

Já Mankiw et al. (1992) interpretam o modelo de Solow tradicionalmente usado para análise de crescimento de maneira diferente de Baumol (1986) e Abramovitz (1986). Segundo os autores, a convergência de renda aconteceria de forma condicional, ou seja, diferentes níveis de steady state seriam encontrados quando houvesse diferenças nos aspectos específicos de cada região, como, por exemplo, diferentes taxas de crescimento populacional e diferentes níveis de capital humano.

Uma questão pertinente à discussão de convergência de renda seriam os aspectos regionais, ou seja, é interessante que se considere a influência dos efeitos espaciais sob a dinâmica de crescimento de cada região. Autores da Nova Geografia Econômica (NGE) como Krugman (1998), por exemplo, conferem ao espaço uma grande importância sobre a determinação do crescimento das regiões. Krugman (1998) argumenta que a aglomeração das atividades em determinado local é explicada por forças que atraem (centrípetas) e que repelem (centrífugas) tais atividades. Assim, essas forças teriam uma forte influência sobre a aglomeração das atividades econômicas em determinadas regiões, aumentando, assim, as suas possibilidades de crescimento econômico. Alguns exemplos de forças centrípetas e centrífugas seriam, respectivamente, facilidades de acesso a mercados e a produtos e altos custos de transporte.

Concernente a esse aspecto, Rey \& Montouri (1998) tratam a convergência regional de renda para os Estados norte-americanos no período 1929-94 sob perspectiva da econometria espacial. Os autores encontram fortes padrões de autocorrelação espacial e evidenciam a existência da má especificação do modelo quando não se leva em conta tais efeitos espaciais.

$\mathrm{O}$ uso de metodologias que consideram a dependência espacial é importante, pois as externalidades de tecnologia existentes entre as regiões afetariam as suas taxas de crescimento. Romer (1986), por exemplo, sugere que o investimento em capital não aumenta somente o nível de tecnologia local, mas também o nível de tecnologia das regiões vizinhas por meio dos transbordamentos de conhecimento. Já Ertur \& Koch (2007) desenvolvem o modelo de Solow espacialmente ampliado. Tal modelo prevê que a taxa de crescimento de determinada região depende das taxas de crescimento dos seus vizinhos, bem como dos transbordamentos de variáveis explicativas, isto é dos transbordamentos espaciais de variáveis como taxa de poupança e nível de capital físico.

Outro aspecto importante relacionado às questões espaciais é a heterogeneidade espacial. Segundo Ertur \& Koch (2007), é improvável assumir que 
os parâmetros que descrevem o crescimento sejam idênticos entre as regiões. Além disso, diversos estudos recentes, como o de Durlauf \& Johnson (1992), sugerem que a premissa de um único modelo linear de crescimento aplicado a todas as economias é incorreta. A grande maioria de trabalhos que analisam a existência de múltiplos equilíbrios consideram a formação ou não de clubes de convergência entre as regiões. Contudo, é interessante verificar a existência de equilíbrios individuais (específicos) para cada região, ou seja, é interessante buscar indicídios de heterogeneidade espacial extrema.

$\mathrm{Na}$ literatura internacional, encontram-se disponíveis dois trabalhos sobre convergência de renda local, utilizando a metodologia de Regressões Ponderadas Geograficamente (RPG). ${ }^{1}$ O primeiro deles foi escrito por Yildirim (2005), que analisa a convergência de renda para as províncias da Turquia entre os anos 1990 e 2001. A análise é dividida em duas etapas: a priori, o autor examina os processos de convergência absoluta e convergência condicional, aplicando modelos econométrico-espaciais que consideram a dependência espacial. Os resultados deste modelo indicam convergência entre as províncias e também indicam a presença de dependência na forma de defasagem espacial. Em um segundo momento, os autores estimam os modelos locais e concluem que os modelos globais podem não capturar os efeitos de algumas variáveis importantes para o crescimento.

Já Wei \& Ie (2009) utilizam a metodologia de RPG para examinar o processo de crescimento da China, especialmente da província de Zhejiang. Os autores comparam os resultados dos modelos locais para os períodos 19901998 e 1998-2004 com os resultados do modelo global estimado por mínimos quadrados ordinários. Os resultados da análise mostram que o uso da RPG traz benefícios ao modelo, melhorando os critérios de informação.

Considerando a heterogeneidade espacial extrema, também se encontra na literatura o trabalho de Ertur \& Koch (2007). Diferente dos trabalhos de Yildirim (2005) e Wei \& Ie (2009) que utilizam a metodologia RPG, Ertur \& Koch (2007) estimam um modelo local, usando o método de estimação local espacial autoregressivo (SALE). A análise é feita para 91 países no período 1960-1995. Os resultados corroboram o modelo teórico desenvolvido pelos autores e indicam a existência tanto de dependência espacial quanto de heterogeneidade. Entretanto, apesar de considerarem os efeitos espaciais, os autores não controlam os efeitos não observados e, portanto, seus resultados poderiam estar inconsistentes.

Já na literatura nacional, não se encontram trabalhos que consideram a heterogeneidade espacial extrema. Entretanto, é importante ressaltar que em um país com "dimensões continentais" como o Brasil, onde as diferenças entre as regiões são tão evidentes, um trabalho sobre crescimento econômico e sobre convergência de renda per capita que não considere tais diferenças, pode apresentar resultados que não sejam robustos e, consequentemente, que não representem bem a realidade.

Dessa forma, a fim de controlar para tanto a dependência espacial quanto a heterogeneidade espacial, o presente trabalho desenvolverá modelos locais de convergência de renda, baseados no modelo de Solow espacialmente ampliado desenvolvido por Ertur \& Koch (2007).

Nesse sentido, serão investigadas as seguintes hipóteses: i) a priori, serão analisados os processos de convergência através de modelos clássicos de re-

\footnotetext{
${ }^{1}$ Os detalhes sobre essa metodologia encontram-se na seção 3.
} 
gressão linear (MCRL) e de primeiras diferenças (DIF), na tentativa de verificar se as características específicas das áreas são relevantes para o processo de crescimento; ii) em um segundo momento, serão verificadas a existência do transbordamento espacial da renda per capita, bem como da dependência espacial; e iii) por fim, será testada a hipótese de que modelos locais, que considerem a especificidade de cada AMC, representem melhor a dinâmica de crescimento das áreas mínimas comparáveis brasileiras.

Um fato importante quando se trata de análise de convergência de renda no Brasil é a diferença do número de municípios ao longo do tempo. Os municípios brasileiros que em 1970 totalizavam 3952, em 2000 totalizaram 5507. Essas alterações territorais, derivadas da emancipação de vários municípios, dificultam a análise dos processos de crescimento municipais. Dessa forma, o uso das áreas mínimas comparáveis (AMCs) se justifica, pois permite a análise de um período mais longo, dado que esse conceito, desenvolvido pelo Instituto Nemesis a partir de informações do IBGE, uniformiza as fronteiras de uma região. ${ }^{2}$ Ao utilizar esse conceito, portanto, é possível fazer uma comparação entre essas áreas sem que haja perda de informações. Em termos de definição, pode-se dizer que as AMCs são as áreas geográficas mais desagregadas que podem ser comparadas ao longo do tempo (Magalhães \& Miranda 2009).

Assim, o presente trabalho é dividido em quatro seções, além dessa introdução. A seguir, a seção 2 elenca alguns dos trabalhos nacionais sobre convergência de renda. A seção 3 apresenta o modelo teórico de Ertur e Koch (2007), as metodologias que serão utilizadas e a descrição das variáveis. A seção 4 apresenta e discute os resultados. Por fim, as considerações finais são reunidas na seção 5 .

\section{Desigualdade de Renda no Brasil e a Literatura Nacional sobre Convergência de Renda}

A análise de convergência de renda para o Brasil é de suma importância já que, apesar desse país ser um dos "campeões" no quesito desigualdade de renda no mundo, alguns trabalhos, como o de Silveira Neto \& Azzoni (2008), apontam uma redução dessa desigualdade nos últimos anos. Entretanto, é possível observar no gráfico 2.1 que, apesar da média do PIB per capita total (em relação a todas as AMCs) ter aumentado entre 1980 e 2007, a diferença entre o PIB per capita das regiões mais ricas (SE e S) e o PIB per capita das regiões mais pobres ( $\mathrm{N}$ e $\mathrm{NE}$ ), tornou-se ainda maior, sugerindo a não existência de um processo de convergência de renda para as regiões brasileiras. Nesse sentido, surge a pergunta: estariam as áreas mínimas comparáveis brasileiras convergindo para um mesmo nível de renda ou será que cada uma das AMCs estaria convergindo para um específico nível de renda?

$\mathrm{Na}$ literatura brasileira encontram-se diversos trabalhos referentes à convergência de renda, entretanto, poucos deles analisam todas as áreas mínimas comparáveis ou todos os municípios brasileiros conjuntamente. Entretanto, a maioria desses trabalhos não controla para as questões espaciais. ${ }^{3}$ Já alguns

\footnotetext{
${ }^{2}$ Esse trabalho irá se referir a municípios e áreas mínimas comparáveis como sinônimos.

${ }^{3}$ Em relação aos estados brasileiros e que não consideram as questões espaciais como dependência espacial e heterogeneidade espacial encontram-se: Ferreira \& Ellery Jr. (1996), Azzoni (1997), Azzoni et al. (2000), Pôrto Jr. \& Ribeiro (2000), Silveira Neto \& Azzoni (n.d.), Souza \&
} 
estudos como o de Pimentel \& Haddad (2004) e Sartoris Neto \& Carvalho (2009) analisam a convergência para municípios ou microrregiões brasileiras corrigindo para a dependência espacial ${ }^{4}$.

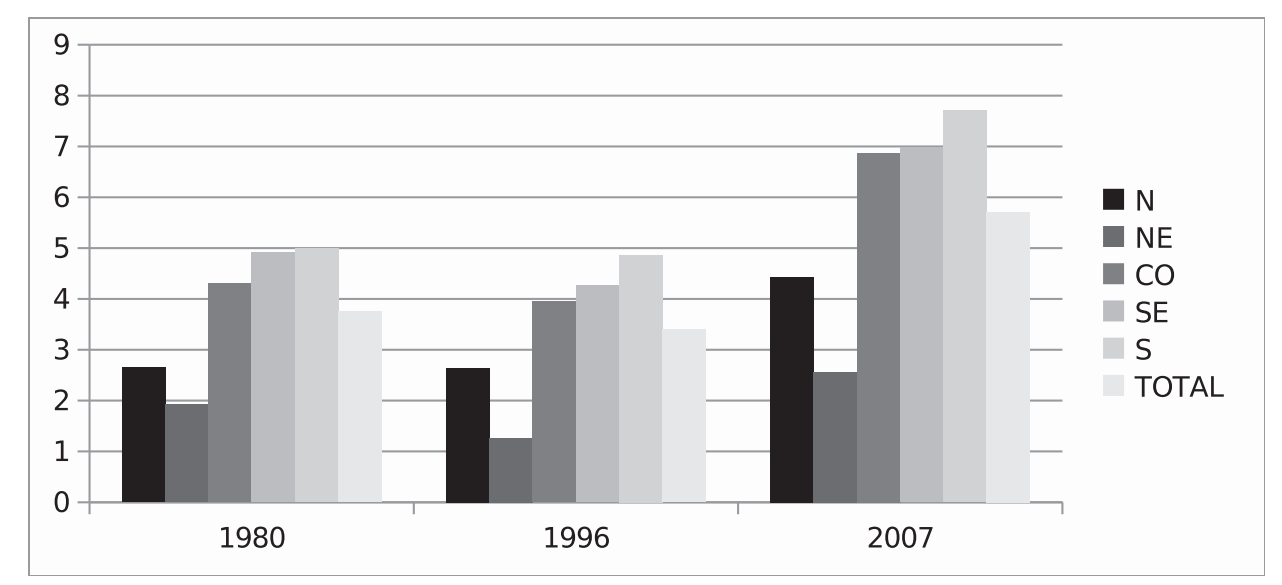

Fonte: Elaboração própria.

Figura 1: PIBs per capita dos estados brasileiros no período 1980/2007

Obs: i) $\mathrm{N}, \mathrm{NE}, \mathrm{CO}, \mathrm{SE}$ e $\mathrm{S}$, indicam, respectivamente, as regiões Norte, Nordeste, Centro-oeste, Sudeste e Sul; ii) A unidade do PIB per capita é reais de 2000 (mil).

Uma pergunta que pode surgir nesse ponto é: por que, apesar de tamanha desigualdade entre os municípios brasileiros, diversos estudos apontam a existência de convergência de renda entre os mesmos? Uma das possíveis explicações para isso repousa na chamada Falácia de Galton. Uma relação negativa entre a renda per capita e a taxa de crescimento encontrada por meio de uma análise em cross section pode não significar convergência de renda de fato, mas sim uma regressão à média.

Além disso, tanto a metodologia de cross sections, quanto de dados em painel, podem gerar resultados não muito robustos, por não considerarem as diferenças existentes entre as regiões. Para controlar o problema a heterogeneidade, alguns estudos como o de Andrade et al. (2007) e Gondim et al. (2007) testam a formação de clubes de converência entre os municípios brasileiros. ${ }^{5}$ No geral, os artigos encontram evidências de divergência quando todos os municípios são analisados em conjunto. Além disso, os resultados principais dos mesmos sugerem a formação de dois clubes de convergência, um mais pobre formado pelos municípios das regiões norte e nordeste e um mais rico formado pelas regiões sul, sudeste e centro-oeste.

Para as áreas mínimas brasileiras (AMCs) é possível encontrar os trabalhos de Coelho \& Figueiredo (2007) e Santolin \& De Figueiredo (2010). Coelho \&

Pôrto Jr. (2002), Cravo \& Soukiazis (2006), Trompieri Neto et al. (2009), Gonçalves et al. (2010). Já com abordagem econométrica-espacial pode-se destacar Magalhães et al. (2000), Magalhães (2001), Barreto \& Almeida (2008), Silveira Neto \& Azzoni (2008). Para os municípios e microrregiões brasileiras, se destacam Alves \& Fontes (2000), Silva et al. (2004), Pinto Coelho (2006), Salvato et al. (2006), Grolli et al. (2006), Fochezzato \& Stulp (2008), Silva Jr. et al. (2008), Ferreira \& Cruz (2008).

${ }^{4}$ Outras estudos que consideram a dependência espacial: Resende (2005), Silva \& Resende (2006), Perobelli et al. (2007), Barreto (2007), Rocha et al. (2008)

${ }^{5}$ Além desses estudos, Laurini et al. (2005), Laurini (2007) e Magalhães \& Miranda (2009), também analisam a formação de clubes de convergência entre os municípios brasileiros. 
Figueiredo (2007) analisam qual das hipóteses de convergência (absoluta, condicional e de clubes) representaria melhor o processo de crescimento econômico das AMCs brasileiras no período 1970/2000. Os autores encontram que o processo de clubes seria o mais ajustado e, portanto, tanto as características estruturais quanto as condições iniciais seriam importantes para a dinâmica de crescimento.

Já Santolin \& De Figueiredo (2010) verifica o efeito da desigualdade interpessoal de renda sobre a taxa de crescimento econômico no período 1970 a 2000, classificando as AMCs quanto aos seus níveis de renda per capita. Dessa forma, na análise foram utilizados quatro grupos: winning, falling behind, catching-up e losing. Tais grupos foram divididos de acordo com a as suas rendas per capita médias e suas taxas de crescimento econômico. Os resultados encontrados sugerem múltiplos estados estacionários e, ademais, sugerem a existência de transbordamentos espaciais de tecnologia (através de efeitos significativos da renda per capita das áreas vizinhas).

Assim, pode-se observar nenhum dos trabalhos acima leva em consideração a heterogeneidade extrema ${ }^{6}$ e, consequentemente, não consideram a hipótese de que possam existir dinâmicas de crescimento econômico específicas a cada região. Além disso, nenhum dos trabalhos acima controla simultaneamente as questões espaciais (dependência e heterogeneidade) e a heterogeneidade não observável e, portanto, seus resultados tendem a estar inconsistentes.

A análise de convergência para o Brasil será referente ao período de 1980 a 2007 e será utilizado o conceito de Áreas Mínimas Comparáveis (AMC) desenvolvido pelo Instituto Brasileiro de Geografia e Estatística (IBGE).

\section{Aspectos Teóricos e Metodológicos}

\subsection{Modelo de Solow Espacialmente Ampliado}

Ertur \& Koch (2007) utilizam o modelo de Solow como base para desenvolver um modelo de crescimento que considera a interdependência tecnológica entre as regiões. Assim como Solow (1956), Ertur \& Koch (2007) usam a seguinte função de produção Cobb-Douglas:

$$
Y_{i}(t)=A_{i}(t) K_{i}(t)^{\alpha} L_{i}(t)^{1-\alpha}
$$

em que as variáveis recebem a notação padrão: $Y_{i}(t)$ é o produto, $K_{i}(t)$ é o nível de capital físico, $L_{i}(t)$ é o nível de trabalho e $A_{i}(t)$ é o nível agregado de tecnologia:

$$
A_{i}(t)=\Omega(t) k_{i}^{\phi}(t) \Pi_{j \neq i}^{N} A_{j}^{\gamma w_{i j}}(t)
$$

Segundo os autores, esta fórmula descreve o nível agregado de tecnologia $A_{i(t)}$ de uma região $i$ como sendo dependente de três termos. O primeiro, $\Omega(t)$, descreve parte do progresso tecnológico como exógeno e idêntico entre as regiões, assim como no modelo de Solow. Assim, $\Omega(t)=\Omega(0) e^{\mu t}$, onde $\mu$ é

\footnotetext{
${ }^{6}$ Entende-se nesse artigo como heterogeneidade extrema as características específicas a cada região. Diferente dos estudos que tratam a heterogeneidade por meio da análise em clubes, a RPG permite analisar o processo de crescimento econômico para cada um dos muncípios brasileiros, encontrando um beta para cada área.
} 
uma taxa de crescimento constante. O segundo termo $k_{i}^{\phi}(t)$ sugere que o nível de tecnologia é crescente com o estoque de capital físico por trabalhador $\left(k_{i}\right)$ disponível na região em questão. O parâmetro $\phi$, com $0<\phi>1$, representa a força das externalidades domésticas geradas pelo acúmulo de capital físico. Já o terceiro termo, representado pelo produtório $\Pi_{j \neq i}^{N} A_{j}^{\gamma w_{i j}}(t)$, diz respeito às externalidades espaciais da tecnologia. Os autores pressupõem a existência de transbordamentos de conhecimento entre regiões vizinhas, entretanto, argumentam que é razoável supor que tais efeitos diminuem a intensidade, com o aumento da distância entre as regiões, dadas as dissimilaridades socioeconômicas e institucionais das regiões. A forma funcional particular assumida por este termo para uma região $i$ é a média ponderada geometricamente, do estoque de conhecimento dos vizinhos, denotado por $j$. O grau interdependência entre as regiões gerado pelo nível de externalidades espaciais é descrito por $\gamma$, onde $0<\gamma<1$. Assume-se que esse parâmetro é igual para todas as regiões, entretanto os efeitos das externalidades espaciais sobre o nível de produtividade da região $i$ dependem da conectividade entre essa região e os seus vizinhos. Ertur \& Koch (2007) sugerem que essa conectividade seja representada pelo termo exógeno $w_{i j}$, para $j=1, \ldots, N$ e $j \neq i$. Quanto maior a conectividade da região $i$ com os seus vizinhos, maiores os benefícios que $i$ obtém das externalidades espaciais. Assim, a principal diferença entre o modelo de Solow e o modelo de Solow espacialmente ampliado se configura no fato de que o estoque de tecnologia de uma região depende da tecnlogia de seus vizinhos. Consequentemente, a renda de uma região dependerá tanto do seu estoque de tecnologia quanto do estoque de seus vizinhos.

Para determinar a equação que descreve a renda por trabalhador da região $i$ no estado estacionário, Ertur \& Koch (2007) reescrevem a função de produção na forma matricial: $y=A+\alpha k$ e, após alguns algebrismos, obtêm:

$$
y=\Omega+(\alpha+\phi) k-\alpha \gamma W k+\gamma W y
$$

De acordo com os autores, quando $\gamma=0$ e $\phi=0$, o modelo passa a ser igual ao modelo de Solow. Reescrevendo esta equação para a economia $i$ e introduzindo a equação da razão capital-produto $\left(K^{*} / Y^{*}\right)$ no estado estacionário em logaritmos, tem-se:

$$
\begin{aligned}
\ln \gamma_{i}^{*}(t)= & \frac{1}{1-\alpha-\phi} \ln \Omega(t)+\frac{\alpha+\phi}{1-\alpha-\phi} \ln s_{i}-\frac{\alpha+\phi}{1-\alpha-\phi} \ln \left(n_{i}+g+\delta\right) \\
& \frac{\alpha \gamma}{1-\alpha-\phi} \sum_{j \neq i}^{N} w_{i j} \ln s_{j}+\frac{\alpha \gamma}{1-\alpha-\phi} \sum_{j \neq i}^{N} w_{i j} \ln \left(n_{j}+g+\delta\right)+ \\
& \frac{\gamma(1-\alpha)}{1-\alpha-\phi} \sum_{j \neq i}^{N} w_{i j} \ln y_{j}^{*}(t)
\end{aligned}
$$

Dessa forma, o modelo de Solow espacialmente ampliado prevê que a renda por trabalhador depende positivamente da taxa de poupança dos seus vizinhos e negativamente das taxas de crescimento da população dos vizinhos.

Segundo Ertur \& Koch (2007), o principal elemento deste resultado é o retorno decrescente do capital. De fato, $\partial\left(k_{i}(t)\right) / \partial k_{i}(t)<0$ desde que $u_{i i}<0$. Em outras palavras, quando uma região aumenta o seu nível de capital físico por 
trabalhador, a taxa de crescimento cai e converge para o seu nível de estado de estacionário. Contudo, um aumento no capital físico por trabalhador das regiões vizinhas, aumenta a produtividade da região $i$ por causa da interdependência tecnológica. Cabe ressaltar que as externalidades do capital físico e a interdependência tecnológica somente retardam o decrescimento da produtividade do capital físico, portanto, o resultado de convergência continua válido sob a hipótese $\alpha+\frac{\emptyset}{1-\gamma}<1$.

Por fim, segundo Ertur \& Koch (2007), somente pode-se dizer que a taxa de crescimento da renda real por trabalhador é uma função negativa da renda por trabalhador inicial depois de controlar para os outros determinantes no estado estacionário. Em outras palavras, a taxa de crescimento da renda real por trabalhador também depende de outros fatores, como a taxa de poupança e o crescimento da população.

\subsection{Metodologias}

Regressões Ponderadas Geograficamente (RPG)

A principal vantagem do modelo de Regressões Ponderadas Geograficamente é a estimação de um modelo específico para cada região, controlando, portanto, para a heterogeneidade espacial extrema. Por meio dessa metodologia é possível analisar a existência do processo de convergência de renda para cada área mínima comparável (AMC), ou seja, é possível especificar o coeficiente beta, a velocidade de convergência e a meia-vida específicos para cada AMC. Ademais, essa metodologia permite o controle da dependência espacial (nas formas de defasagem espacial, de erro e de transbordamentos da variável explicativa) e também permite o controle de efeitos não observados por meio de primeiras diferenças. ${ }^{7}$

A metolodogia de Regressões Ponderadas Geograficamente (RPG) foi desenvolvida por Fotherigan et al. (2002). Tal metodologia se baseia na análise de regressão tradicional, entretanto estima coeficientes locais para cada região (Fotherigan et al. 2002).

O modelo de RPG estima uma equação para cada localidade, usando subamostras dos dados. As regiões que fazem parte destas sub-amostras são escolhidas de acordo com as suas distâncias em relação ao local para o qual está sendo calculada a regressão (Fotherigan et al. 2002). Este modelo propõe a estimação de uma regressão para cada região e, além disso, assim como a Lei de Tobler (1970), admite que dados mais próximos exerçam maior influência do que dados relativos às áreas mais distantes. Dessa forma, o modelo RPG propõe a seguinte equação 5 :

$$
y_{i}=\beta_{0}\left(u_{i}, v_{i}\right)+\sum_{k} \beta_{k}\left(u_{i}, v_{i}\right) x_{i k}+\varepsilon_{i}
$$

onde $\left(u_{i}, v_{i}\right)$ indica as coordenadas do ponto i no espaço, $\beta_{k}\left(u_{i}, v_{i}\right)$ é uma função contínua no ponto $i$ e $x_{i k}$ são as variáveis explicativas de cada região $i$, sendo $k$ igual ao número de variáveis independentes para cada região.

O estimador de RPG é baseado no método de mínimos quadrados ordinários e é calculado da seguinte forma:

\footnotetext{
${ }^{7}$ Para mais informações, ver Wooldridge (2002).
} 


$$
\beta\left(u_{i}, v_{i}\right)=\left(X^{\prime} W\left(u_{i}, v_{i}\right) X^{-1} X^{\prime} W\left(u_{i}, v_{i}\right) y\right.
$$

onde $W$ é uma matriz de ponderação fixa ou adaptativa, em que $w_{i j}$ indicam os pesos baseados na distância entre a observação no ponto $i$ e as demais observações da sub-amostra selecionada pela função do kernel espacial.

A estimação de $\beta\left(u_{i}, v_{i}\right)$, feita por mínimos quadrados ponderados, tem os seus pesos modificados sob influência da proximidade com o ponto de regressão $i$. Esta ponderação é feita pela função kernel espacial. A largura da banda é um dos pontos importantes desse método, sendo que essa largura pode ser constante (constituindo um kernel espacial fixo) e adaptativa. O kernel adaptativo, que será o método utilizado neste trabalho, possui uma vantagem sobre o fixo: a largura da sua banda se adapta ao número de observações em torno do ponto a ser observado, obtendo estimações mais eficientes e menos enviesadas. No caso do kernel adaptativo, nas áreas onde as observações são mais escassas, o kernel se expande e, quando as observações são mais abundantes o kernel se encolhe.

A metodologia de RPG permite, portanto, controlar o problema da heterogeneidade extrema. Entende-se nesse artigo como heterogeneidade extrema as características específicas a cada região. Diferente dos estudos que tratam a heterogeneidade por meio da análise em clubes, a RPG permite analisar o processo de crescimento econômico para cada um dos muncípios brasileiros, encontrando um beta para cada área.

A metodologia até aqui representada busca tratar a heterogeneidade espacial, dada pelas diferenças dos coeficientes entre as regiões. Na tentativa de controlar a dependência espacial, o presente trabalho se propõe a construir modelos locais de defasagem espacial (SAR), de erro autorregressivo (SEM), de defasagem com erro autorregressivo (SAC), o regressivo cruzado espacial (SLX), o de Durbin Espacial (SDM), bem como o modelo de Durbin com erro autorregressivo (SDEM).

\section{Modelo RPG com Dependência Espacial}

O RPG possibilita modelar a dependência espacial na sua forma extrema. Quando o crescimento de uma região é influenciado pelo crescimento de regiões próximas, é interessante incorporar no modelo a variável dependente defasada espacialmente, da seguinte forma:

$$
y_{i}=\alpha\left(u_{i}, v_{i}\right)+\rho\left(u_{i}, v_{i}\right) W y_{i}+\sum_{k} \beta_{k}\left(u_{i}, v_{i}\right) X_{i k}+\varepsilon_{i}
$$

onde $W y$ é a variável dependente defasada espacialmente através de uma matriz de pesos espaciais que será definida mais a frente e $\rho$ define o grau de autocorrelação espacial. Esse modelo é chamado modelo de Defasagem Espacial (SAR). Cabe salientar que para expurgar a endogeneidade da variável $W y$ em relação à $y$, o modelo é estimado por variáveis instrumentais, tendo como instrumentos as variáveis $W X$. Em outras palavras, estima-se uma regressão auxiliar de $W y$ contra as variáveis explicativas, $\mathrm{X}$, e contra as suas defasagens espaciais, $W X$. Depois, utiliza-se o valor predito de $W y$ na regressão 7.

A metodologia RPG permite ainda a modelagem do SEM local, que visa modelar a interação ocorrida através dos efeitos não-modelados, e do SLX local que tem como objetivo de investigar os transbordamentos existentes nas 
variáveis explicativas do modelo. Tais modelos são representados, respectivamente, da seguinte forma:

$$
\begin{aligned}
& y_{i}=\alpha\left(u_{i}, v_{i}\right)+\sum_{k} \beta_{k}\left(u_{i}, v_{i}\right) X_{i k}+\varepsilon \\
& \varepsilon=\lambda\left(u_{i}, v_{i}\right) W_{\varepsilon}+\xi
\end{aligned}
$$

e

$$
y_{i}=\alpha\left(u_{i}, v_{i}\right)+\sum_{k} \tau_{k} W x_{i k}+\sum_{k} \beta_{k}\left(u_{i}, v_{i}\right) X_{i k}+\varepsilon_{i}
$$

Além disso, serão construídos modelos SDM e SDEM locais. O SDM incorpora a idéia do transbordamento por meio da defasagem das variáveis explicativas $(W X)$ e também inclui variável endógena defasada $(W y)$. Este modelo pode ser representado matematicamente da seguinte forma:

$$
y_{i}=\alpha\left(u_{i}, v_{i}\right)+\rho\left(u_{i}, v_{i}\right) W y_{i}+\sum_{k} \beta_{k}\left(u_{i}, v_{i}\right) X_{i k}+\sum_{k} \tau_{k} W x_{i k}+\varepsilon_{i}
$$

De maneira semelhante ao modelo SAR, para expurgar a endogeneidade existente entre $W y$ e $y$, o modelo SDM é estimado pelo método de variáveis instrumentais. Assim, as variáveis contidas na matriz $W X$ são usadas como instrumentos para as próprias variáveis $W X$ e para instrumentalizar $W y$ são utilizadas as defasagens espaciais das defasagens espaciais de $X$, ou seja, $W W X$.

Já o modelo SDEM local, além de incluir as defasagens das variáveis explicativas $(W X)$, também considera que o erro espacial segue um processo de média móvel de primeira ordem, sendo o modelo especificado da seguinte forma:

$$
\begin{aligned}
y_{i} & =\alpha\left(u_{i}, v_{i}\right)+\sum_{k} \beta_{k}\left(u_{i}, v_{i}\right) X_{i k}+\sum_{k} \tau_{k} W x_{i k}+\varepsilon_{i} \\
\varepsilon_{i} & =\lambda\left(u_{i}, v_{i}\right) W \varepsilon+\xi
\end{aligned}
$$

Por fim, cabe salientar que os modelos SEM e SDEM são estimados por meio do método de Kelejian \& Prucha (1999), que, para se garantir a consistência desses estimadores, não necessita que erros sejam normais. O estimador de Kelejian e Prucha é obtido através do seguinte procedimento:

1. Estima-se o modelo (11) por MQO para se obter as estimativas de $\beta$ e $\lambda$.

2. Obtêm-se os resíduos da estimação do modelo da seguinte forma:

$$
\hat{\varepsilon}=y-X \hat{\beta}
$$

3. Com a adoção de um procedimento à la Cochrane-Orcutt, transformamse as variáveis do modelo por intermédio da filtragem espacial, do seguinte modo: $y *=y-\lambda W y$ e $X *=X-\lambda W X$, onde $y *$ e $X *$ indicam as variáveis filtradas.

Dessa forma, este trabalho tentará eliminar: a heterogeneidade não observada através do método de Primeiras Diferenças, a heterogeneidade espacial extrema com o auxílio do RPG e, por fim, a autocorrelação espacial com o auxílio dos modelos locais SAR, SEM, SLX, SDM e SDEM. 


\subsection{Especificação Empírica}

Para a construção do modelo empírico baseado no modelo de Solow espacialmente ampliado sem a consideração de efeitos espaciais, utiliza-se a seguinte especificação:

$$
C R E S_{i t}=\beta_{0}+\beta_{1} P I B i_{i t-1}+c_{i}+\varepsilon_{i t}
$$

onde CRES $S_{i t}$ é a variável dependente "taxa de crescimento do PIB per capita", $P I B i_{i t}$ corresponde ao PIB inicial da área mínima comparável $i$ no período $t$.

\section{Descrição das variáveis}

A análise do presente trabalho concerne a 3658 áreas mínimas comparáveis (AMCs) no período 1980/2007. As variáveis dependente e explicativas serão utilizadas em diferenças, com o objetivo de controlar os efeitos não observados, fixos e constantes. ${ }^{8}$ Para essa análise são utilizadas as variáveis "taxa de crescimento do PIB per capita" (CRES) e "PIB per capita inicial" (PIBi). A construção dessas variáveis é feita da seguinte forma:

1. Variável dependente $\left(C R E S_{i t}\right)$ : A variável dependente em estudo é a taxa de crescimento do PIB per capita das áreas mínimas comparáveis brasileiras. Para a construção dessa variável foram utilizados dados do Instituto Brasileiro de Geografia e Estatística (IBGE), referentes ao PIB municipal e às estimativas de população residente em cada município. A construção da variável é feita da seguinte forma: $C R E S_{i t}=\ln \left(P I B_{i t} / P I B_{i t-1}\right)$.

2. Variável explicativa "PIB per capita inicial" (PIBi): O nível de renda per capita inicial é representado pela variável logaritmo natural do PIB per capita da AMC corrigido a preços de 2000, obtidos da base de dados do Sistema de Contas Nacionais do IBGE. O nível de renda per capita inicial é incluído para testar a hipótese de convergência de renda, ou seja, de que quanto maior o PIB per capita inicial da região, menor a sua taxa de crescimento. Além disso, por intermédio da interpretação de seu coeficiente é possível verificar a velocidade da convergência e a meia-vida.

A Tabela 1 apresenta as estatísticas descritivas das variáveis utilizadas na análise. As taxas de crescimento das áreas mínimas comparáveis referem-se aos períodos 1980/1996 e 1996/2007. Como se pode observar, em média, as AMCs brasileiras cresceram cerca de $23 \%$. Já em relação à variável PIB per capita inicial (PIBi), pode-se observar que a mediana é bem menor que a média, indicando uma grande desigualdade do PIB per capita, dado que a maioria das AMCs apresentam PIB per capita inferior à média.

\section{Resultados}

Nessa seção são apresentados os resultados dos modelos estimados para as áreas mínimas comparáveis no período 1980/2007. Seguiremos o seguinte

\footnotetext{
${ }^{8} \mathrm{O}$ método de primeiras diferenças é usado quando a análise é feita para dois períodos, onde os efeitos não observados são eliminados através da transformação de primeiras diferenças. Para maiores detalhes ver Wooldridge (2002).
} 
Tabela 1: Estatísticas Descritivas das Variáveis

\begin{tabular}{lcccrrc}
\hline Variável & Média & Mediana & Desvio-padrão & Mínimo & Máximo & N $^{\circ}$ de obs. \\
\hline CRES & 0.230 & 0.236 & 0.567 & -3.282 & 3.914 & 7318 \\
PIBi & 3.312 & 2.502 & 6.400 & 0.046 & 455.915 & 7318 \\
\hline
\end{tabular}

Fonte: Elaboração própria com base nos dados do IBGE.

procedimento: em um primeiro momento, estima-se o modelo básico e verifica-se a presença de dependência espacial nos resíduos. Caso não haja dependência, encerra-se o procedimento e considera-se o modelo básico como o mais apropriado. Caso contrário, estimam-se os modelos de dependência espacial (SLX, SAR, SEM e SDM e SDEM) e, por meio da qualidade das estimações e do critério de informação Akaike (AIC), determina-se o melhor modelo.

A priori, são apresentadas na Tabela 2 as estimações referentes ao modelo clássico de regressão linear (MCRL) e ao modelo de primeiras diferenças (DIF). A estimação "MCRL" é relativa ao período 1980/2007, já "DIF" corresponde à primeira diferença entre os períodos 1980/1996 e 1996/2007.

Tabela 2: Resultados Globais dos Modelos Clássico de Regressão Linear (MCRL) e de Primeiras Diferenças (DIF)

\begin{tabular}{lcc}
\hline Variáveis & MCRL & DIF \\
\hline Constante & $0.762^{* * *}$ & $0.542^{* * *}$ \\
PIBi & $69.3377^{* * *}$ & 80.718 \\
& $-0.335^{* * *}$ & $-1.282^{-37.099}$ \\
\hline R2 ajustado & 0.27 & 0.71 \\
AIC & 4489.40 & 3597.55 \\
SC & 4501.81 & 3609.96 \\
\hline Multicolinearidade & 2.59 & 1.27 \\
Jarque-Bera & $6119.67^{* * *}$ & $2234.90^{* * *}$ \\
Koenker-Basset & $17.03^{* * *}$ & $177.56^{* * *}$ \\
\hline I de Moran & $14.49^{* * *}$ & $13.68^{* * *}$ \\
ML (erro) & $209.39^{* * *}$ & $186.72^{* * *}$ \\
ML (erro) robusto & $279.62^{* * *}$ & $123.11^{* * *}$ \\
ML (defasagem) & $106.18^{* * *}$ & $65.97^{* * *}$ \\
ML (defasagem) robusto & $176.41^{* * *}$ & 2.36 \\
\hline No de obs. & 3659 & 7318 \\
\hline
\end{tabular}

Fonte: Elaboração própria com base nos dados do IBGE.

Obs: i) Os valores em negrito indicam os valores da estatística $t$; ii) Para a estimação dos diagnósticos de autocorrelação espacial, foi utilizada a matriz de um vizinho mais próximo $(\mathrm{K} 1)$; e iii) ${ }^{*},{ }^{* *} \mathrm{e}^{* * *}$ indicam que os coeficientes são significativos aos níveis de 1,5 e $10 \%$, respectivamente.

É importante frisar que, ao contrário do modelo (DIF), o modelo MCRL não considera os efeitos fixos. Ao analisar os modelos MCRL e DIF, observamos que em ambos o coeficiente da variável PIB per capita (PIBi) inicial é negativo e significativo, o que indica um processo de convergência. Contudo, quando os efeitos fixos são considerados (modelo DIF), o impacto de PIBi 
sobre a taxa de crescimento é bem maior, passando de -0,335 (no modelo MCRL) para -1,282 (no modelo DIF). Tal resultado sugere que ao considerar aspectos não observados e constantes (como por exemplo, cultura, valores e instituições de cada região) a força (e conequentemente, a velocidade) de convergência aumenta. Os critérios de informação Akaike (AIC) e Schwarz (SC) apontam uma melhora no modelo quando os efeitos fixos são considerados (modelo DIF). Isso sugere que considerar aspectos não observados e constantes é de suma importância para a determinação do processo de crescimento econômico local.

É interessante observar uma diferença básica nos resultados dos modelos MCRL e DIF. Apesar de ambos apresentarem um coeficiente negativo e significativo estatiscamente e utilizarem em sua regressão as mesmas variáveis, somente os resultados do MCRL indicam um processo de convergência absoluta. Ou seja, os municípios estariam convergindo para uma mesma renda de equilíbrio. Contudo, o coeficiente $\beta$ do modelo DIF sugere a existência de convergência condicional: os municípios só convergeriam para a mesma renda caso apresentassem características não observadas idênticas. Dada a diversidade existente entre os municípios brasileiros, supõem-se que alguns destes poderiam convergir a rendas semelhantes, todavia, é uma hipótese muito forte supor que poderiam convergir para a mesma renda, uma vez que cultura, valores e instituição são características peculiares de cada localidade.

A autocorrelação espacial pode ser observada na última parte da Tabela 2. O valor significativo e positivo do teste global I de Moran sugere a presença de autocorrelação espacial nos resíduos de ambos os modelos (MCRL e DIF), assim os dados tendem a estar concentrados no espaço. Apesar de fornecer essa informação, o teste é incapaz de sugerir o tipo de dependência existente (se dependência na forma de erro autorregressivo ou se na forma de defasagem espacial, por exemplo). Assim sendo, torna-se interessante também a análise dos testes específicos do tipo multiplicador de Lagrange (ML). Segundo tais diagnósticos, pode-se observar a presença de dependência espacial tanto na forma de erro, quanto na forma de defasagem espacial no MCRL. Contudo, ao considerarmos os efeitos das variáveis não observadas (modelo DIF), os testes de autocorrelação passam a indicar somente a presença de dependência na forma de erro, pois a estatística ML robusta da defasagem não é significativa. Para o MCRL é possível perceber tanto a dependência espacial na forma de erro quanto na forma da defasagem espacial, uma vez que todas as estatísticas são significativas. Contudo, quando analisamos o modelo DIF, a não significância da estatística ML robusta da defasagem nos indica a presença somente da dependência na forma de erro. Tais resultados relativos ao modelo DIF podem ser entendidos da seguinte forma: as variáveis não observadas incluídas no termo de erro tendem a estar concentradas no espaço e a transbordar entre as regiões. Assim, variáveis como, por exemplo, capital humano e nível de investimento de uma região estariam afetando o crescimento econômico das regiões vizinhas.

Na tentativa de corrigir o problema da dependência espacial são estimados os seguintes modelos: de Defasagem Espacial (SAR), de Durbin Espacial (SDM), Regressivo Cruzado Espacial (SLX), de Erro Auto-regressivo (SEM) e de Durbin com Erro Auto-regressivo (SDEM). Todos os modelos são estimados usando a matriz de variância-covariância consistente de White para corrigir o problema da heterocedasticidade. 


\subsection{Modelos de Dependência Espacial para o Período Compreendido Entre 1980 e 2007}

Nessa seção, serão apresentados os modelos de dependência espacial para a primeira diferença feita entre os períodos 1980/1996 e 1996/2007. Os resultados desses modelos estão reportados na Tabela 3.

Os vários modelos (SAR, SEM, SDM, SLX e SDEM) foram estimados com o objetivos de se encontrar o modelo mais ajustado de acordo com os critérios de informação AIC e SC e que corrija o problema da autocorrelação espacial. Esses critérios apontam que o modelo que melhor representa o processo de crescimento econômico entre as AMCs é o SDEM, uma vez apresentam os menores valores em comparação ao outros modelos (iguais a, respectivamente, 3190, 91 e 3209,53). O modelo considera a existência de dependência da forma de erro (como sugerido pela estatística ML do erro) e também de transbordamentos espaciais do PIB per capita inicial. Ao averiguar os resíduos desse modelo quanto à persistência da dependência espacial, foi encontrado um valor não significativo estatisticamente para estatística $I$ de Moran e, portanto, não se pôde rejeitar a hipótese nula de aleatoriedade dos dados. ${ }^{9}$

O valor significativo e positivo da variável defasada espacialmente do PIB per capita inicial indica que a renda das áreas vizinhas afeta positivamente o crescimento econômico de determinada área mínima. Em outras palavras, a proximidade geográfica com municípios de renda mais elevada gera benefícios para a localidade. Diferente dos modelos clássicos de regressão linear, onde o impacto de uma variável pode ser dado pelo seu coeficiente, o impacto marginal total da variável explicativa PIBi sobre a taxa de crescimento é dado pela soma dos seus efeitos direto $(\beta)$ mais indireto $(\tau)$ (Lesage \& Pace 2009). Assim, o impacto total de PIBi sobre a variável dependente é igual a $-1,156$ $(-1,299+0,043)$. sugerindo a existência de um processo de convergência de renda condicional. Os resultados indicam, portanto, que as áreas mínimas só convergiriam para a mesma renda (reduzindo totalmente a desigualdade) caso apresentassem as mesmas características não observadas e os mesmos efeitos espaciais decorrentes do processo de dependência espacial. Pode-se dizer que o processo de convergência de renda nesse caso seria dependente de uma semelhança dessas determinadas características iniciais de cada município. O impacto positivo da defasagem espacial do PIB inicial per capita (PIBi) sugere que, durante o processo de crescimento econômico, ocorrem externalidades tecnológicas positivas (Ertur \& Koch 2007). Quanto ao coeficente relativo aos transbordamentos do erro, a sua significância sugere que as variáveis incluídas no termo de erro de uma região afetam o processo crescimento de seus vizinhos. Tais variáveis (como, por exemplo, capital humano) poderiam estar desencadeando esse efeito de transbordamento entre as regiões.

Para a correção da heterogeneidade espacial extrema foi estimado o modelo de coeficientes locais por RPG. Em outras palavras, foram estimados um modelo para cada município, controlando o problema da heterogeneidade extrema. Ao comparar o critério de informação AIC dos modelos global e local, encontra-se m valor mais baixo para o modelo local (igual a 2728.46), em comparação ao modelo de coeficientes globais $(3190,91)$. Essa melhora no critério sugere que a estimação de um modelo que considera a existência de múltiplos equilíbrios é mais ajustada à análise do processo de crescimento econômico

\footnotetext{
K1.

${ }^{9} \mathrm{O}$ z-value computado pela estatística I de Moran é igual a 0.353 , tendo como base a matriz
} 
Tabela 3: Resultados dos Modelos Espaciais para o Período 1980/2007

\begin{tabular}{|c|c|c|c|c|c|}
\hline Variáveis & SAR & SDM & SLX & SEM & SDEM \\
\hline Constante & $0.542^{* * *}$ & $0.553^{* * *}$ & $0.546^{* * *}$ & $0.366^{* * *}$ & $0.370^{* * *}$ \\
\hline PIBi & $-1_{0.021}^{0.007}$ & $-1.295^{* * *}$ & $\begin{array}{l}-1.290^{* * *} \\
-56.923\end{array}$ & $\begin{array}{l} \\
-1.31040^{* * *} \\
-57.951\end{array}$ & $\begin{array}{l}-1.299^{* * *} \\
-55.836\end{array}$ \\
\hline WPIBi & & $\begin{array}{c}0.022 \\
0.03 \\
0.037\end{array}$ & $\begin{array}{c}-56.923 \\
0.046^{* * *}\end{array}$ & & $\begin{array}{c}-55.836 \\
0.043^{\text {*** }} \\
(-4,243)\end{array}$ \\
\hline$\lambda$ (erro) & & & & $0.319^{* * *}$ & $0.319^{* * *}$ \\
\hline$\rho(\operatorname{lag})$ & $\begin{array}{c}1.000^{* * *} \\
0.06\end{array}$ & $\begin{array}{r}-0.012 \\
0.027 \\
\end{array}$ & & & \\
\hline AIC & 3191.93 & 3590.24 & 3588.25 & 3198.95 & 3190.91 \\
\hline SC & 3210.54 & 3615.06 & 3606.86 & 3211.36 & 3209.53 \\
\hline $\mathrm{N}^{\mathrm{o}}$ de obs. & 7318 & 7318 & 7318 & 7318 & 7318 \\
\hline
\end{tabular}

dos municípios brasileiros. Tal resultado justifica-se pela heterogeneidade existente entre as áreas mínimas e pela chamada Falácia de Galton. Resultados semelhantes são encontrados por Laurini et al. (2005) e Magalhães \& Miranda (2009) que também encontram a existência de múltiplos equilíbrios. A diferença aqui se dá pelo fato de que ambos os trabalhos citados encontram a existência de dois clubes de convergência de renda, um formado pelas regiões norte e nordeste e outro formado por centro-oeste, sul e sudeste. Assim, tais trabalham sugerem que os municípios presentes no mesmo clube estariam convergindo para uma mesma renda de equilíbrio, apesar dos dois clubes estarem divergindo em termos de renda. Continuasse, então, considerando que todos os municípios presentes nas regiões sul, sudeste e centro-oeste apresentam um mesmo processo de crescimento econômico, uma hipótese bastante forte, dada a tamanha heterogeneidade entre os municípios dessas regiões. Já a análise por meio da metodologia de RPG apresenta-se como um avanço, pois nos permite dizer que cada região estaria convergindo para a sua própria renda de equilíbrio.

Para analisamos se cada coeficientes varia entre as áreas mínimas, utilizase o este de Monte Carlo (Tabela 4). Este teste, portanto, avalia a instabilidade estrutural dos coeficientes e possui como hipótese nula $(\mathrm{H} 0)$ a estabilidade dos coeficientes.

Tabela 4: Teste de Monte Carlo para o modelo SDEM para a primeira diferença entre 1980/1996 e 1996/2007

\begin{tabular}{ll}
\hline Coeficiente & $\mathrm{p}$-valor \\
\hline Constante & 0 \\
PIBi & 0,01 \\
WPIBi & 0,320 \\
\hline
\end{tabular}

Fonte: Elaboração própria com base nos dados do IBGE.

É interessante notar na Tabela 4 que o coeficiente de WPIBi é estacionário, ou seja, o efeito indireto do PIB per capita inicial é constante em todas as 
AMCs. Dessa forma, pode-se dizer que as externalidades tecnológicas são positivas e afetam igualmente todas as áreas. Ou seja, existem transbordamentos de tecnologia e conhecimento entre as regiões, que ajudam a promover o crescimento econômico. Isso sugere que ao elevar o seu produto interno bruto, uma região produz externalidades positivas que são captadas e absorvidas pelas regiões vizinhas. Resultado semelhante é encontrado por Brasil Gondim et al. (2007) que encontram que a localização das regiões afeta o seu PIB per capita, provavelmente via transbordamentos de conhecimento, efeitos de escala e/ou proximidade entre centros produtores e consumidores.

Já o efeito direto, dado pelos coeficientes significativos de PIBi, é específico para cada AMC. Santolin \& De Figueiredo (2010), ${ }^{10}$ em uma análise de clubes de convergência para as AMCs, também encontram diferentes impactos do PIB per capita inicial sobre sob diferentes distribuições das taxas de crescimento.

A Figura 2 apresenta os impactos totais locais da variável PIBi que são dados pela soma do efeito direto mais o efeito indireto dessa variável. ${ }^{11}$ Os impactos marginais totais são significativos e negativos, o que indica um processo de convergência para todas as AMCs. Contudo, percebe-se que há uma diferença entre a "força" de convergência dessas áreas, em outras palavras, em algumas áreas o PIB per capita inicial (PIBi) apresenta um impacto maior sobre a taxa de crescimento econômico do que em outras. Os resultados sugerem, portanto, que os impactos do PIBi sobre a taxa de crescimento são peculiares a cada município e dependem das características estruturais, iniciais e específicas de cada município. É muito importante ressaltar que as áreas mínimas se encontram divididas em cinco grupos para facilitar a apresentação. A metodologia de Regressões Ponderadas Geograficamente (RPG) não possibilita per si a divisão das regiões em grupos ou "clubes". A metodologia RPG fornece um resultado para cada região e uma vez que os testes apontam os resultados locais mais robustos que os resultados globais, pode-se dizer que cada região estaria convergindo para a sua própria renda de equilíbrio.

As regiões com os maiores valores absolutos de beta, representadas pelos intervalos "alto" e "muito alto", são as áreas que estariam mais próximas da sua renda de equilíbrio. Assim, a maioria dos municípios situados nos estados Espírito Santo (ES), Rio de Janeiro (RJ), parte dos municípios da Bahia (BA), do Amazonas (AM) e do Acre (AC) e da região Sul apresentariam um processo de convergência mais próximo de se finalizar e, consequentemente, as diferenças de renda presentes entre esses municípios permaneceriam inalteradas. Os resultados também apontam que as regiões representadas pelas cores mais claras, classificadas "baixo" e "muito baixo" se encontram mais distantes da sua renda de equilíbrio e, dessa forma, teriam maiores de probabilidades de alcançarem rendas mais altas no longo prazo em comparação com suas rendas atuais. Estas áreas encontram-se basicamente nas regiões Nordeste e CentroOeste e no estado do Pará (PA). Isso faz surgir duas observações. A primeira, em relação à região Centro-Oeste, cujas rendas de seus municípios se configuram entre as maiores do país e que, além disso, já vem há alguns anos apresentando uma tendência significativa de crescimento. Com o seu processo de crescimento econômico ainda relativamente distante de a renda de equilíbrio,

\footnotetext{
${ }^{10}$ Assim como Magalhães \& Miranda (2009) e Laurini (2007).

${ }^{11}$ Cabe salientar que todos os coeficientes da variável PIBi são significativos estatisticamente ao nível de $5 \%$.
} 


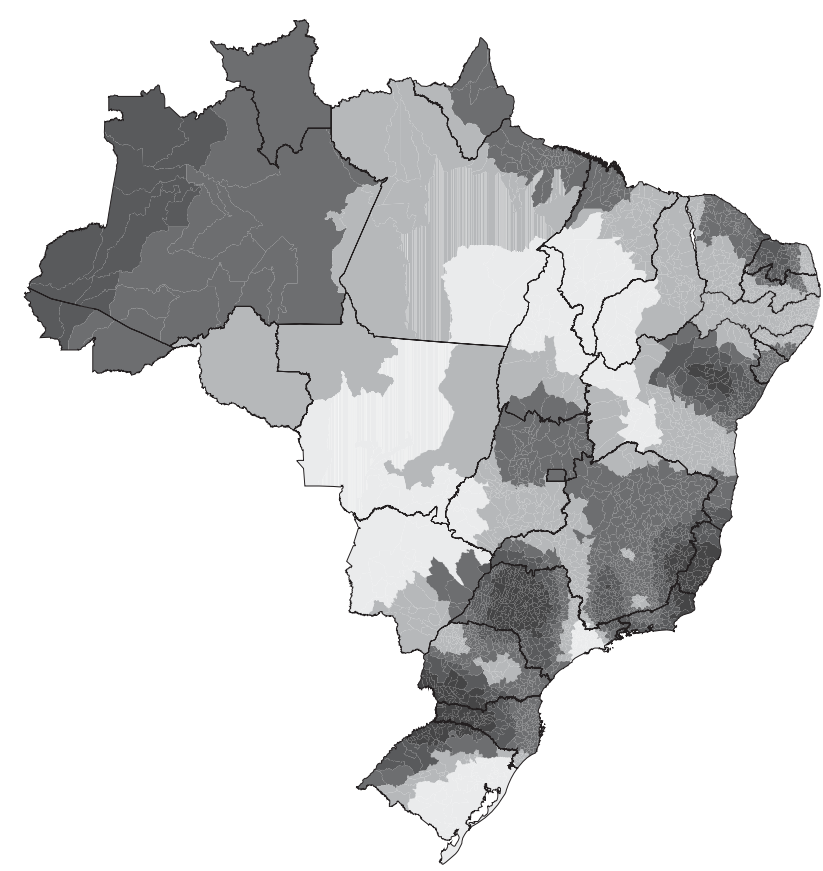

\section{Impactos}

\begin{tabular}{ll} 
& Muito Alto \\
\hline & Alto \\
& Médio \\
& Baixo \\
\hline \hline & Muito Baixo
\end{tabular}

Fonte: Elaboração própria com base nos dados do IBGE.

a Obs: O impacto total da variável PIB per capita inicial é igual pela soma do efeito direto (dado pelo coeficiente de PIBi) mais o efeito indireto (dado pelo coeficiente de WPIBi, ou seja, os transbordamentos localizados).

Figura 2: Impactos Totais Locais referentes à variável PIB per capita inicial (PIBi)

pode-se observar que, no longo prazo, o Centro-Oeste poderia se apresentar como uma região significativamente rica em relação às demais. A segunda observação se faz em relação aos municípios com baixa força de convergência presentes em regiões pobres, como o Nordeste e o Estado do Pará. Os resultados apontam que tais municípios ainda se encontram relativamente distantes da sua renda de equilíbrio, sugerindo que haja a possibilidade de, no longo prazo, tais regiões reduzirem a desigualdade de renda frente aos municípios que fazem parte das regiões mais ricas do país, como os dos estados de São de Paulo e Rio de Janeiro.

No grupo das regiões cujos impactos são classificados como médios, temse basicamente municípios dos estados de Minas Gerais (principalmente do norte do estado) e da região Norte, localizados nos estados do Amazônia, Acre e Roraima. Atenção maior deve ser dada à formulação de políticas públicas voltadas a esses municípios classificados com médio, alto e muito alto, cujo histórico apresenta rendas per capitas bastante baixas, uma vez que, segundo indicam os resultados, tais municípios não estariam tão distantes da sua renda de equilíbrio. Caso o processo de crescimento dessas áreas seja mantido como está, provavelmente, a desigualdade entre suas as rendas e as rendas dos municípios mais ricos irá persistir.

A Figura 2 também sugere que os impactos marginais totais da variável PIBi apresentam um padrão espacial. Para verificar a existência desse padrão é utilizada a estatística $I$ de Moran. Ao computar essa estatística é encontrado um valor para $I$ igual a 0.994 a um nível de significância de $1 \%$, indicando uma forte tendência de concentração dos impactos no espaço. Isso ressalta a importância do espaço e indica que o processo de convergência deve ser se- 
melhante entre vizinhos. AMCs com altos impactos tendem estar localizadas entre vizinhos que também apresentem altos impactos.

Por fim, outro ponto interessante a ser ressaltado são os períodos de meiavida observados. As áreas cujos impactos da variável PIBi são classificados como muito alto apresentam os menores períodos de meia-vida. A recíproca também é verdadeira, ou seja, áreas cujos impactos da variável PIBi são muito baixo apresentam os maiores períodos de meia-vida. Os períodos de meiavida encontrados para as AMCs no período de análise variam entre 18 e 28 anos. Tais períodos (de meia-vida) estão relacionados a velocidades de convergência que variam de 2,46 a 3,67\% e, portanto, são maiores do que a velocidade de convergência encontrada por Barro \& Sala-I-Martin (1995) (igual a 2,2\%). Esse resultado é esperado, pois existiriam menos barreiras para a mobilidade de trabalho e capital dentro de um país do que entre países.

\section{Considerações Finais}

O presente trabalho investigou os processos de convergência absoluta, condicional e local para as áreas mínimas comparáveis (AMCs) brasileiras no período 1980/2007. Ademais, considerou-se também a dependência espacial nas formas de erro, de defasagem e de transbordamentos das variáveis explicativas. Tais análises locais buscaram averiguar a existência de coeficientes $\beta$ e de velocidades de convergência específicos para cada área.

Os principais resultados indicaram que o modelo de primeiras diferenças é capaz de explicar melhor o processo de convergência das AMCs quando comparado ao modelo clássico de regressão linear. Dessa forma, fica explícita a importância dos efeitos não observados no processo de crescimento econômico. Além disso, os resultados também sugeriram a presença de dependência espacial nas formas de erro e de transbordamento da variável explicativa, indicando a importância do espaço na determinação do processo de crescimento econômico.

Os resultados referentes à estimação do modelo local apontaram que, de fato, a heterogeneidade existente entre os municípios brasileiros deve ser levada em consideração na análise do crescimento econômico. Tais resultados sugeriram que cada município estaria convergindo para a sua específica renda de equilíbrio e que, portanto, cada regiao apresentaria um processo de crescimento econômico distinto.

Por fim, se por um lado as diferenças encontradas entre os processos de crescimento dos municípios brasileiros sugerem que a região nordeste, por seus municípios ainda estarem distante das rendas de equilíbrio, apresenta chances de reduzir a desigualdade de sua renda frente aos municípios mais ricos do país, sugerem também que um cuidado maior deve ser tomado em relação à formulação de políticas públicas voltadas para Amazonas, Acre e Roraima, uma vez que estas regiões (que são consideradas relativamente pobres) já estariam mais próximas das suas rendas de equilíbrios.

\section{Referências Bibliográficas}

Abramovitz, M. (1986), 'Catching up, forging ahead, and falling behind', Journal of Economic History 46. 
Alves, R. F. \& Fontes, R. (2000), Clubes de convergência entre os municípios de minas gerais, in 'Seminário sobre Economia Mineira. Anais.'.

Andrade, E., Laurini, R. M. \& Pereira, P. L. V. (2007), 'Convergence clubs among brazilian municipalities', Economics Letters 83, 179-184.

Azzoni, C., Menezes Filho, N., Menezes, T. \& Silveira Neto, R. (2000), 'Geografia e convergência de renda entre os estados brasileiros', IPEA.

Azzoni, C. R. (1997), 'Concentração regional e dispersão das rendas per capita estaduais: análise a partir de séries históricas estaduais de PIB, 19391995', Estudos Econômicos 27(3).

Barreto, R. C. S. (2007), Desenvolvimento Regional e Convergência de Renda nos Municípios do Estado do Ceará, Tese de doutorado, UFV.

Barreto, R. C. S. \& Almeida, E. (2008), A contribuição do capital humano para o crescimento econômico e convergência espacial do pib per capita no ceará, in 'Fórum BNB 2008. Anais'.

Barro, R. \& Sala-I-Martin, X. (1995), Economic Growth. New York: Mc Graw - Hill.

Baumol, W. J. (1986), 'Productivity growth, convergence and welfare: What the long-run data show', American Economic Review 76.

Baumol, W. J. \& Wolff, E. N. (1988), 'Productivity growth, convergence and welfare: Reply', American Economic Review 78(5).

Coelho, R. L. P. \& Figueiredo, L. (2007), 'Uma análise da hipótese de convergência para os municípios brasileiros', RBE 61(3), 331-352.

Cravo, T. \& Soukiazis, E. (2006), 'O capital humano como fator determinante para o processo de convergência entre os estados do brasil', Encontro Regional de Economia/Nordeste: Estratégias de Desenvolvimento Regional, Anais.

Durlauf, S. \& Johnson, P. (1992), Local versus global convergence across national economies, in 'Working paper n. 3996', National Bureau of Economic Researches, Cambrigde.

Ertur, C. \& Koch, W. (2007), 'Growth, technological interdependence and spatial externalities: Theory and evidence', J. Appl Econ 22.

Ferreira, P. \& Ellery Jr., R. (1996), 'Convergência entre a renda per capita dos estados brasileiros', Revista de Econometria .

Ferreira, R. T. \& Cruz, M. A. (2008), 'Clubes de convergência na desigualdade de renda nos municípios brasileiros', Encontro Nacional de Economia, Anais.

Fochezzato, A. \& Stulp, V. J. (2008), 'Análise de convergência de renda per capita entre os municípios do rio grande do sul, 1985 a 1998', Ensaios FEE 29(1).

Fotherigan, A. S., Brundsdon, C. \& Charlton, M. (2002), Geographically Weighted Regressions: the Analysis of Spatially Varying Relationships, Jonh Wiley \& Sons ltd. 
Gondim, J. L. B., Barreto, F. A. \& Carvalho, J. R. (2007), 'Condicionantes de Clubes de Convergência no Brasil', Estudos Econômicos 37, 71-100.

Gonçalves, E., Ribeiro, E. C. B. A. \& Freguglia, R. S. (2010), 'Transbordamentos de conhecimento e capacidade de absorção: Uma análise para os estados brasileiros', XXXVIII Encontro Nacional de Economia - ANPEC. Anais.

Grolli, P. A., Oliveira, C. A. \& Jacinto, P. A. (2006), 'Crescimento econômico e convergência com a utilização de regressões quantílicas: um estudo para os municípios do rio grande do sul (1970 a 2001)', XXXIV Encontro Nacional de Economia - ANPEC. Anais.

Kelejian, H. H. \& Prucha, I. R. (1999), 'A generalized moments estimator for the autorregressive parameter in a spatial model', International Economic Review 40(2).

Krugman, P. (1998), 'What's new about new economic geographic', Oxford Review of Economic Policy 14.

Laurini, M. (2007), 'A note on the use of quantile regression in beta convergence analysis', Economics Bulletin 3, 1-8.

Laurini, M., Andrade, E. \& Pereira, P. L. V. (2005), 'Income convergence clubs for brazilian municipalities:a non-parametric analysis', Applied Economics 37, 2099-2118.

Lesage, J. P. \& Pace, R. K. (2009), Introduction to Spatial Econometrics, CRC Press.

Magalhães, A., Hewings, G. J. D. \& Azzoni, C. R. (2000), 'Spatial dependence and regional convergence in brazil', Working Paper REAL. 00-T-11, Urbana Chanpaign.

Magalhães, A. M. (2001), 'Clubes de convergência no brasil: uma abordagem com correção espacial', Encontro Nacional de Economia - ANPEC. Anais.

Magalhães, J. C. R. \& Miranda, R. B. (2009), 'Dinâmica da renda per capita, longevidade e educação nos municípios brasileiros', Estudos Econômicos 39(2), 539-569.

Mankiw, N., Romer, D. \& Weil, D. (1992), 'A contribution to the empirics of economic growth', The Quarterly Journal of Economics 107, 407-437.

Perobelli, F. S., Faria, W. R. \& Ferreira, P. G. (2007), 'Análise da Convergência Espacial do PIB per capita no estado de Minas Gerais', Revista Brasileira de Estudos Regionais e Urbanos 1, 85-113.

Pimentel, E. A. \& Haddad, E. A. (2004), 'Análise da distribuição espacial da renda dos municípios de minas gerais: uma abordagem setorial', Texto para discussão, NEREUS.

Pinto Coelho, R. L. (2006), Dois Ensaios sobre a Desigualdade de Renda dos Municípios Brasileiros, Dissertação de mestrado, Centro de Desenvolvimento e Planejamento Regional - Faculdade de Ciências Econômicas UFMG. 
Pôrto Jr., S. S. \& Ribeiro, E. P. (2000), 'Dinâmica de crescimento regional uma análise empírica para a região sul', Revista Econômica do Nordeste 31.

Resende, G. M. (2005), Teste de Robustez e Externalidades Espaciais: o caso dos estados brasileiros e dos municípios mineiros, Dissertação de mestrado, Centro de Desenvolvimento e Planejamento Regional - Faculdade de Ciências Econômicas - UFMG.

Rey, S. J. \& Montouri, B. D. (1998), 'Us regional income convergence: A spatial econometric perspective', Regional Studies 33(2), 143-156.

Rocha, L. E., Cardoso, D. F. \& Rodrigues, L. (2008), 'Índice de desenvolvimento da família (idf) e a convergência de renda: uma análise espacial para os municípios do estado de minas gerais, 1991 a 2000', XXXVI Encontro Nacional de Economia. Anais.

Romer, P. (1986), 'Increasing returns and long run growth', Journal of Political Economy.

Salvato, M. A., Raad, R. J., Araújo Jr., A. F. \& Pessoa, F. M. (2006), 'Disparidades regionais em minas gerais', XI Seminário sobre Economia Mineira. Anais.

Santolin, R. \& De Figueiredo, L. (2010), 'Desigualdade interpessoal de renda: Implicações sobre o crescimento econômico dos municípios brasileiros', Encontro Nacional de Economia, Anais.

Sartoris Neto, A. \& Carvalho, A. C. (2009), 'Crescimento econômico e externalidades espaciais nos municípios do estado do pará: 1991 e 2000', VII Encontro Nacional da ABER. Anais.

Silva, A. M. \& Resende, G. M. (2006), Crescimento econômico comparado dos municípios alagoanos e mineiros: uma análise espacial, in 'Texto para discussão', number 1162, Brasília.

Silva, E., Fontes, R. \& Alves, L. F. (2004), 'Análise das disparidades regionais em minas gerais', XI Seminário Sobre a Economia Mineira. Anais.

Silva Jr., G. G., Viana, G. I., Oliveira, J. M. \& Abreu, E. A. P. (2008), 'Uma análise comparativa da convergência entre as rendas per capitas dos municípios de alagoas nos anos de 1995 e 2005', VI Encontro Nacional da ABER, Anais.

Silveira Neto, R. M. \& Azzoni, C. R. (2008), 'Non-spatial policies and regional income inequality in brazil', RSAI Congress. Anais.

Silveira Neto, R. M. \& Azzoni, C. R. (n.d.), 'Radiografando a convergência regional: fontes setoriais e mudanças estruturais.'.

URL: Disponivel em: http://www.nemesis.org.br/download.php

Solow, R. (1956), 'A contribution to the theory of economic growth', Quartely Journal of Economics 98.

Souza, N. J. \& Pôrto Jr., S. S. (2002), 'Crescimento regional e novos testes de convergência para os municípios da região nordeste do brasil'.

URL: Disponivel em: www.ufrgs.br/ppge/pcientifica/2002_11.pdf 
Tobler, W. R. (1970), 'A computer movie simulating urban growth in the detroit region', Economic Geography.

Trompieri Neto, N., Castelar, I. \& Linhares, F. C. (2009), 'Convergência de renda dos estados brasileiros: uma abordagem de painel dinâmico com efeito threshold', Encontro Nacional de Economia, Anais.

Wei, I. H. D. \& Ie, X. (2009), 'Beyond convergence: Space, scale and regional inequality in china', Tijdchroft voor Economische en Sociale Geografie 10(1).

Wooldridge, J. M. (2002), Econometric Analysis of Cross Section and Panel Data, MIT Press.

Yildirim, J. (2005), 'Regional policy and economic convergence in turkey: a spatial data analysis', 18th European Advanced Studies - Institute in Regional Science, Anais. 